\title{
Effect of Body Habitus and Heart Rate on Accuracy of Aortic-Radial Transfer Functions for Predicting Central Hemodynamic Indices in Growing Children
}

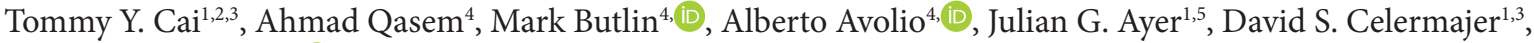 \\ Michael R. Skilton ${ }^{1,2, *}$, (D) \\ ${ }^{1}$ Sydney Medical School, The University of Sydney, Sydney, NSW, Australia \\ ${ }^{2}$ Boden Collaboration for Obesity, Nutrition, Exercise and Eating, The University of Sydney, Sydney, NSW, Australia \\ ${ }^{3}$ Department of Cardiology, Royal Prince Alfred Hospital, Sydney, NSW, Australia \\ ${ }^{4}$ Australian School of Advanced Medicine, Macquarie University, Sydney, NSW, Australia \\ ${ }^{5}$ Heart Centre for Children, The Children's Hospital at Westmead, Sydney, NSW, Australia
}

\section{ARTICLE INFO}

Article History

Received 25 May 2020

Accepted 21 September 2020

\section{Keywords}

Central blood pressure central arterial hemodynamics transfer functions paediatrics

\begin{abstract}
Central Systolic Blood Pressure (cSBP) and Central Augmentation Index (cAIx) have been independently associated with cardiovascular risk in adults, and can be estimated using a generalized peripheral-to-central transfer function. We hypothesize that transfer function accuracy and precision will be influenced by degree of growth and change in body habitus during periods of growth, such as in childhood. We studied the pulse pressure waveforms of 91 healthy children at 8 -years of age, and subsequently at 14 -years of age. Waveforms were obtained by high-fidelity applanation tonometry of the radial and carotid arteries. Individualized radial-to-carotid transfer functions were formulated at 8-years of age and applied at 14-years of age to estimate cSBP and cAIx. Accuracy was defined as the difference between directly measured and predicted values, and precision as the residuals. Changes in weight (vs. error: $\beta=-0.22, p=0.04$; vs. residuals: $\beta=0.30 ; p<0.01$ ) and body mass index (vs. residuals: $\beta=0.28, p<0.01$ ), between 8 and 14 years of age were associated with accuracy and precision of individualized transfer functions in predicting cSBP at 14 years of age. Change in weight (vs. residuals: $\beta=0.30 ; p<0.01$ ) and heart rate (vs. residuals $\beta=0.32, p<0.01$ ) were associated with the precision in predicting cAIx. Changes in body habitus and heart rate over a 6-year period of growth are associated with the predictive accuracy and precision of individualized transfer functions in children suggestive of changes in the frequency response characteristics of an individual's vascular system during growth periods.
\end{abstract}

(c) 2020 Association for Research into Arterial Structure and Physiology. Publishing services by Atlantis Press International B.V. This is an open access article distributed under the CC BY-NC 4.0 license (http://creativecommons.org/licenses/by-nc/4.0/).

\section{INTRODUCTION}

High blood pressure is an important cardiovascular risk factor and a major cause of morbidity and mortality worldwide [1]. Systolic Blood Pressure (SBP) tracks strongly from childhood to adulthood, and early life exposures can influence later adult hypertension and other aspects of cardiovascular health [2,3]. For example, it has been demonstrated that fetal growth restriction and premature birth is associated with elevated blood pressure in adulthood, with similar findings independently associating lower birth-weight with adult coronary artery disease and diabetes $[4,5]$.

Guidelines for treatment of hypertension are based largely around measurements of Peripheral SBP (pSBP), although central blood pressure, particularly in the aorta, more accurately reflects the pressure against which the left ventricle heart must pump. Indeed, central arterial hemodynamic indices, such as Central SBP (cSBP) and Central Augmentation Index (cAIx), have previously been

"Corresponding author.Email: michael.skilton@sydney.edu.au

Peer review under responsibility of the Association for Research into Arterial Structure and Physiology

Data availability statement: Participant raw waveform data used in this study may be available on reasonable request to the authors. associated with increased cardiovascular risk and adverse outcomes in adults, independent of peripheral blood pressure [6-8]. In contrast, data from the Framingham Heart Study demonstrated no significant incremental improvement in predictive cardiovascular modelling using central hemodynamics parameters over peripheral blood pressure values [9]. The true prognostic value of central arterial hemodynamics, and along with it the means to accurately estimating central blood pressure, remains under investigation.

Central arterial waveforms can be estimated non-invasively from the combination of peripheral blood pressure and peripheral pulse waveform by use of a Transfer Function (TF), or directly measured by tonometry of the centrally located arteries such as the carotid artery [10-12]. TFs allow waveforms from one part of the vascular system to be mathematically transformed into a waveform at another location within the system, most commonly from a peripheral artery with a readily accessible pulse such as the radial artery to a central artery, such as the ascending aorta. From these synthesized central waveforms, central hemodynamic indices such as cSBP and cAIx can then be extracted. Generalized TFs (gTF) are derived from ensemble-averaged Individualized TFs (iTF), and are widely used in adult hemodynamic research and have an emerging 
role in clinical practice, particularly in the diagnosis of borderline hypertension and guidance of antihypertensive therapy [13-15].

It has been postulated that the anatomy of the vascular tree, such as branch points, are a major feature determining the modulus of iTFs in adults, and that ensemble-averaging of iTFs across individuals to generate a gTF is reasonable given that there is minimal change in these properties with age and disease within an individual, and sufficient similarity between adult individuals $[10,16]$. Nevertheless, given that iTFs directly model the frequency response characteristics of an individual's vascular system, they should better model an individual's vascular pulse wave dynamics than a gTF [17], and may better predict changes in an individual's central blood pressure over time within the context of a static vascular system. We hypothesized that iTFs developed during childhood would be less accurate at a later age after a period of considerable growth, and that the degree of predictive error may be directly associated with one or more features of physical or physiological change between these two ages.

Accordingly, we sought to generate iTFs for children at 8 years, and subsequently apply these iTFs to the same individuals 6 years later at 14 years of age to determine their accuracy. We then sought to determine the factors most strongly associated with error in the estimated central pressures.

\section{MATERIALS AND METHODS}

\subsection{Participants}

The Childhood Asthma Prevention Study (CAPS) was a randomized controlled trial of healthy 8-year old children allocated to omega-3 fatty acid supplementation and house-dust mite avoidance (in a $2 \times 2$ factorial design) from birth to 5 -years of age, to determine the effect on the incidence of asthma and atopy in children at risk of these conditions [18]. A cardiovascular substudy (CardioCAPS) was undertaken in 405 CAPS participants without diabetes who attended the 8-year follow-up and agreed to participate in further testing of the determinants of cardiovascular disease in children $(n=269)$. One hundred and eighty three CAPS participants who followed up at 14-years of age also consented to participate in the CardioCAPS substudy.

This study was approved by the human research ethics committees of the University of Sydney, the Children's Hospital at Westmead, and Sydney South West Area Health Service. Written informed consent was obtained from the parent or legal guardian of each participating child.

\subsection{Anthropometric Data}

Height, weight, and waist circumference were measured for each participant at both 8- and 14-years of age, and Body Mass Index (BMI) calculated.

\subsection{Peripheral and Central Pressure Waveform Data}

Peripheral and central pressure waveform data was obtained non-invasively at both 8 - and 14-years of age. All subjects were examined in a supine position in a quiet room. pSBP and Diastolic Blood Pressures
(DBP) were obtained using a validated automated oscillometric device (Welch Allyn Vital Signs Monitor; Welch Allyn, NY, USA) [19]. Peripheral and central arterial pressure waveforms were derived by high-fidelity applanation tonometry from arterial pulse waveforms taken consecutively from the radial and left common carotid artery (SPC-301, Millar Instruments; TX, USA), and processed with waveform analysis software using a previously validated method (Sphygmocor CvMS version 9, AtCor Medical; Sydney, Australia) [20]. All data were recorded in continuous sequences of 10 pulses. Peripheral and carotid waveforms were acquired in atotal 269 subjects at 8-years of age, and 183 subjects at 14-years of age. Resting Heart Rate (HR) was obtained separately as part of the tonometry data set.

\subsection{Subject Waveform Selection and Screening}

For each individual at each age of visit we selected waveform data that were within $5 \mathrm{bpm}$ of one another for the purposes of waveform averaging to reduce noise from excessive heart rate variation between recordings. Only participant data sets with complete and accurately recorded data at both 8- and 14-years of age were included. This yielded a total of 97 study participants with paired waveform data. Data were reviewed prior to statistical analyses with incorrectly entered or incomplete data removed (i.e. misrecorded data) prior to statistical analysis $(n=93)$ (Figure S1).

\subsection{Peripheral and Central Waveform Processing}

Waveform data were processed separately to produce averaged waveforms for each participant at each age (8- and 14-years). Waveform data was initially processed using a signal filter (Savitsky-Golay: Labview 12.0, National Instruments Corporation; TX, USA) followed by identification of individual cycles, while iteratively screening out cycles with abnormal lengths (Mean \pm 2 SD) and trends ( $>20 \%$ absolute difference between starting and ending amplitudes). Next, the included cycles were detrended (linearly smoothing out differences between starting and ending cycle amplitudes). Furthermore, data were aligned in time such that the rising component of the pressure waveform coincided with the rising component of the averaged waveform, and the individual waveforms whose mean error was greater than two times the average mean error of the averaged waveform were excluded. Remaining data were averaged and truncated in order to unify length of central and peripheral waveforms. Finally, waveform amplitudes were calibrated according to measured DBP and calculated Mean Arterial Pressure (MAP). MAP was calculated by integration of the radial waveform calibrated to pSBP and DBP. For clarification, we refer to central hemodynamic indices measured from waveforms obtained from carotid tonometry as directly 'measured' and indices measured from iTF-derived waveforms as 'estimated' (Figure S2).

\subsection{Production and Application of iTFs}

Individualized transfer functions were produced using the 8-year waveform data from all 95 subjects using an Autoregressive with Extra Input (ARX) linear parametric model as described previously [10]. In the ARX model, the peripheral waveform was designated as 
the input and the central waveform as the output. The model orders for the ARX model were chosen to minimize mean squared error between the peripheral waveform-dependent predicted waveform and measured central waveforms. iTFs produced using the data from the 8-year study visit were applied to peripheral waveforms from the same participant at 14 years of age to estimate central hemodynamic indices.

Central systolic blood pressure was defined as the maximum of central pulse pressure waveforms, and cAIx as the ratio of the augmentation pressure to pulse pressure, where the augmentation pressure is the difference between the second and first systolic peaks. cSBP and cAIx was calculated from processed waveforms using the Sphygomocor CvMS system (version 9, AtCor Medical).

\subsection{Statistical Analysis}

Statistical analysis was undertaken using IBM SPSS Statistics (version 21, IBM Corporation; NY, USA). Data are presented as mean and Standard Deviation (SD). Bivariate Pearson correlation was used to assess linear association between variables. Statistical significance was inferred at $2 p \leq 0.05$. Plots were created in Prism (version 7, Graphpad; San Diego, USA). Bland-Altman analyses and modified Bland-Altman plots were used to describe accuracy and precision of iTFs at 8- and 14-years of age. Predictive error and residual sum of squares of predictive error of iTF-derived central hemodynamic indices were used to reflect accuracy and precision, respectively.

\section{RESULTS}

\subsection{Participant Characteristics}

Participant characteristics at ages 8 and 14 years are described in Table 1. Height, weight, BMI, waist circumference, brachial pSBP, $\mathrm{DBP}$ and MAP increased between ages 8 and 14 years, while resting HR decreased. $\operatorname{pSBP}(\beta=0.08, p=0.46)$, measured $\operatorname{cSBP}(\beta=-0.05$, $p=0.62)$ and estimated $\operatorname{cSBP}(\beta=0.04, p=0.67)$ were not associated with height at 8 years of age. Contrastingly, $\operatorname{pSBP}(\beta=0.24$, $p=0.02)$, but not measured $\operatorname{cSBP}(\beta=0.15, p=0.15)$ or estimated $\operatorname{cSBP}(\beta=0.17, p=0.11)$ was associated with height at 14 years of age in these same children. Split by sex, boys gained more height $(\Delta=0.46 \mathrm{~m}, p<0.01)$ and less BMI $\left(\Delta=-1.1 \mathrm{~kg} / \mathrm{m}^{2}, p<0.01\right)$ between the two ages than girls, but not weight $(\Delta=-1.1 \mathrm{~kg}$, $p=0.59)$ or $\operatorname{HR}(\Delta=-3.1 / \mathrm{s}, p=0.10)$. There was no significant difference in change in pSBP between boys and girls, nor were there any significant differences between iTF accuracy or precision in estimating cSBP between boys and girls.

\subsection{Determinants of Changes in pSBP, cSBP and cAlx}

Table 2 shows the associations of changes in pSBP, directly measured cSBP and cAIx from 8- to 14-years of age with concurrent changes in anthropometric measures and HR. Changes in pSBP and cSBP were both directly associated with an increase in weight, BMI and waist circumference, but not height. cSBP and cAIx, but not pSBP, was inversely associated with resting HR.

\section{3. cSBP and cAlx by Individualized Transfer Function Modelling}

The iTF-estimated cSBP and cAIx at 8- and 14-years of age, and the values measured directly from the carotid artery are shown in Table 3. At 8 years of age, the age at which the iTFs were formulated, there is a small mean difference between estimated and measured CSBP and CAIx, demonstrating the expected accuracy of the iTFs when applied to the cohort in which they were derived. Although there is a marked increase in predictive error at 14-years of age, the predictive accuracy of the iTFs are

Table 1 Participant characteristics

\begin{tabular}{lccc}
\hline $\boldsymbol{n}=\mathbf{9 3}$ & At 8 years old & At $\mathbf{1 4}$ years old & Difference \\
\hline Sex, male & $57 \%$ & - & - \\
Height, cm & $128(5)$ & $163(7)$ & $36^{*}$ \\
Weight, kg & $28(5)$ & $58(13)$ & 30 \\
BMI, kg/m ${ }^{2}$ & $17.1(2.2)$ & $21.6(4.3)$ & $4.5^{*}$ \\
Waist circumference, cm & $59(6)$ & $73(11)$ & 15 \\
pSBP, mmHg & $100(7)$ & $115(9)$ & 14 \\
DBP, mmHg & $58(5)$ & $65(6)$ & 6 \\
MAP, mmHg & $74(5)$ & $80(7)$ & 6 \\
Resting HR, bpm & $80(8)$ & $72(9)$ & -9 \\
Measured cSBP, mmHg & $92(8)$ & $104(12)$ & 11 \\
Measured cAIx, \% & $-15(10)$ & $-28(13)$ & -12 \\
\hline
\end{tabular}

"Indicates a significant difference in change between boys and girls. Data presented as Mean (SD).

Table 2 Pearson correlation of changes in pSBP and directly measured central hemodynamic indices with concurrent changes in participant characteristics between 8 and 14 years

\begin{tabular}{|c|c|c|c|c|c|c|}
\hline & \multicolumn{2}{|l|}{$\Delta \mathrm{pSBP}$} & \multicolumn{2}{|l|}{$\Delta \mathrm{cSBP}^{*}$} & \multicolumn{2}{|l|}{$\Delta \mathrm{cAIx}^{*}$} \\
\hline & $\begin{array}{l}\text { Pearson correlation } \\
\text { coefficient }\end{array}$ & $p$-value & $\begin{array}{l}\text { Pearson correlation } \\
\text { coefficient }\end{array}$ & $p$-value & $\begin{array}{l}\text { Pearson correlation } \\
\text { coefficient }\end{array}$ & $p$-value \\
\hline$\Delta$ Height, $\mathrm{m}$ & 0.02 & 0.84 & -0.09 & 0.40 & -0.19 & 0.08 \\
\hline$\Delta$ Weight, kg & 0.26 & 0.01 & 0.21 & 0.04 & 0.03 & 0.76 \\
\hline$\Delta \mathrm{BMI}, \mathrm{kg} / \mathrm{m}^{2}$ & 0.27 & 0.01 & 0.21 & 0.05 & 0.09 & 0.40 \\
\hline$\Delta$ Waist circumference, $\mathrm{cm}$ & 0.32 & $<0.01$ & 0.23 & 0.03 & 0.09 & 0.40 \\
\hline$\Delta$ Resting HR, bpm & 0.17 & 0.10 & 0.23 & 0.03 & -0.23 & 0.03 \\
\hline
\end{tabular}

"Central SBP and AIx as measured from direct carotid tonometry. 
Table 3 Measured versus iTF-derived central SBP and AIx at 8 and 14 years of age, with comparison to results obtained from using an age-appropriate paediatric gTF

\begin{tabular}{|c|c|c|c|c|}
\hline & Measured & iTF-estimated & Difference & Difference $^{* *}$ \\
\hline \multicolumn{5}{|c|}{8 years assessment } \\
\hline Central SBP, $\mathrm{mmHg}^{*}$ & $92.3(7.5)$ & $92.2(7.2)$ & $-0.1(1.0)$ & $2.1(4.4)$ \\
\hline Central AIx, \%* & $-15.8(10.1)$ & $-16.8(10.5)$ & $-0.9(4.6)$ & $-6.6(11.9)$ \\
\hline \multicolumn{5}{|c|}{14 years assessment } \\
\hline Central SBP, $\mathrm{mmHg}^{*}$ & $103.6(11.5)$ & $105.1(11.0)$ & $1.5(6.7)$ & $4.9(6.1)$ \\
\hline Central AIx, \%* & $-27.8(13.1)$ & $-32.1(17.4)$ & $-5.2(19.8)$ & $-8.4(18.0)$ \\
\hline
\end{tabular}

*Results presented as Mean (SD). "Difference between central hemodynamic indices estimated using a previously developed generalized an age-appropriate paediatric gTF and directly measured carotid waveform.

comparable to previously published values using an ageappropriate generalized paediatric TF (Table 3). Predictive error between estimated and directly measured values for cSBP and cAIx increased to $1.5 \pm 6.7 \mathrm{mmHg}$ and decreased to $-5.2 \pm 19.8 \%$ respectively (Figures S3 and S4). Although there is an increase in error measuring cSBP using the iTF at 14 years of age compared to 8 years, estimates would be considered accurate at both ages as per the ARTERY society recommendations which accept validation cut-offs for central blood pressure devices within $5 \pm 8 \mathrm{mmHg}$ [21]. There are no similar criteria to judge absolute accuracy for measuring cAIx estimates.

\subsection{Effect of Height on Modelling Accuracy of Individualized Transfer Function at 14 Years of Age}

The relationship between change in height between 8- and 14-years of age, and the accuracy and precision of iTFs in estimating cSBP and cAIx at 14 years of age is shown in Figure 1. Change in height was not associated with accuracy $(\beta=0.05, p=0.65)$ nor precision $(\beta=0.13, p=0.22)$ of estimated cSBP, nor with the accuracy $(\beta=0.10, p=0.40)$ or precision $(\beta=0.11, p=0.89)$ of estimated cAIx. Associations with change in height were not affected by sex.

\subsection{Effect of Weight on Modelling Accuracy of Individualized Transfer Function at 14 Years of Age}

The associations between change in weight between age 8- and 14-years of age, and the accuracy and precision of iTFs in estimating CSBP and cAIx at 14 years of age are shown in Figure 2. Change in weight is associated with accuracy $(\beta=-0.22, p=0.04)$ and precision $(\beta=0.30, p<0.01)$ of estimated cSBP. For estimated cAIx, change in weight is associated with precision $(\beta=0.30, p<0.01)$, but not accuracy $(\beta=0.13, p=0.25)$. When split by sex, change in weight was associated with accuracy of estimated cSBP for boys $(\beta=-0.51, p<0.01)$ but not for girls $(\beta=0.25, p=0.11)$, while associated with precision of estimated $\operatorname{cSBP}$ for girls $(\beta=0.37$, $p=0.02)$ but not for boys $(\beta=0.25, p=0.08)$. Similarly, change in weight was associated with both accuracy and precision of estimated cAIx for boys (accuracy: $\beta=0.34, p=0.03$; precision: $\beta=0.36, p=0.02$ ), but not for girls (accuracy: $\beta=-0.41, p=0.81$; precision: $\beta=0.07, p=0.70$ ).

\subsection{BMI and Accuracy of Individualized Transfer Functions at 14 Years of Age}

The relationship between change in BMI between 8- and 14-years of age, and the accuracy and precision of iTFs in estimating cSBP and cAIx at 14 years of age was also investigated (Figure S5). Change in BMI was not associated with accuracy of estimated cSBP $(\beta=-0.13, p=0.21)$, but was associated with precision $(\beta=0.28$, $p<0.01)$. However, when split by sex, change in BMI was associated with accuracy and precision of cSBP estimation in boys (accuracy: $\beta=0.37, p=0.02$; precision: $\beta=0.31, p=0.05$ ), but only accuracy in girls (accuracy: $\beta=-0.27, p=0.05$; precision: $\beta=0.26, p=0.07$ ). Change in BMI was not associated with either accuracy of estimated cAIx $(\beta=0.05, p=0.96)$ nor precision $(\beta=0.10, p=0.39)$, and this was not influenced by sex. The associations of change in waist circumference between 8 - and 14-years of age with the accuracy and precision of estimated CSBP and cAIx at 14 years of age were similar (Figure S6).

\subsection{HR on Modelling Accuracy of Individualized Transfer Function at 14 Years of Age}

The relationship between change in HR between 8- and 14-years of age, and the predictive error of estimated cSBP and cAIx at 14 years of age is shown in Figure S7. Change in HR is not associated with either accuracy of cSBP estimates $(\beta=-0.16$, $p=0.13)$ nor precision $(\beta=-0.11, p=0.31)$, although when split by sex, change in HR is associated with precision of cSBP estimates in boys only $(\beta=-0.28, p=0.05)$. Change in $\mathrm{HR}$ is not associated with predictive accuracy of cAIx $(\beta=0.08, p=0.51)$ but is associated with precision of cAIx estimates $(\beta=0.32, p<0.01)$. When split by sex, this remained the case only for precision of cAIx estimates in boys $(\beta=-0.46, p<0.01)$.

\section{DISCUSSION}

In this study, we used individualized radial-to-carotid pulse waveform TFs to assess whether a 6-year period of extensive physical and physiological change during childhood affects the accuracy and precision of vascular property modelling. Our findings suggest that changes in HR and body size, in particular measures of adiposity, may have a meaningful effect on the underlying hemodynamic 

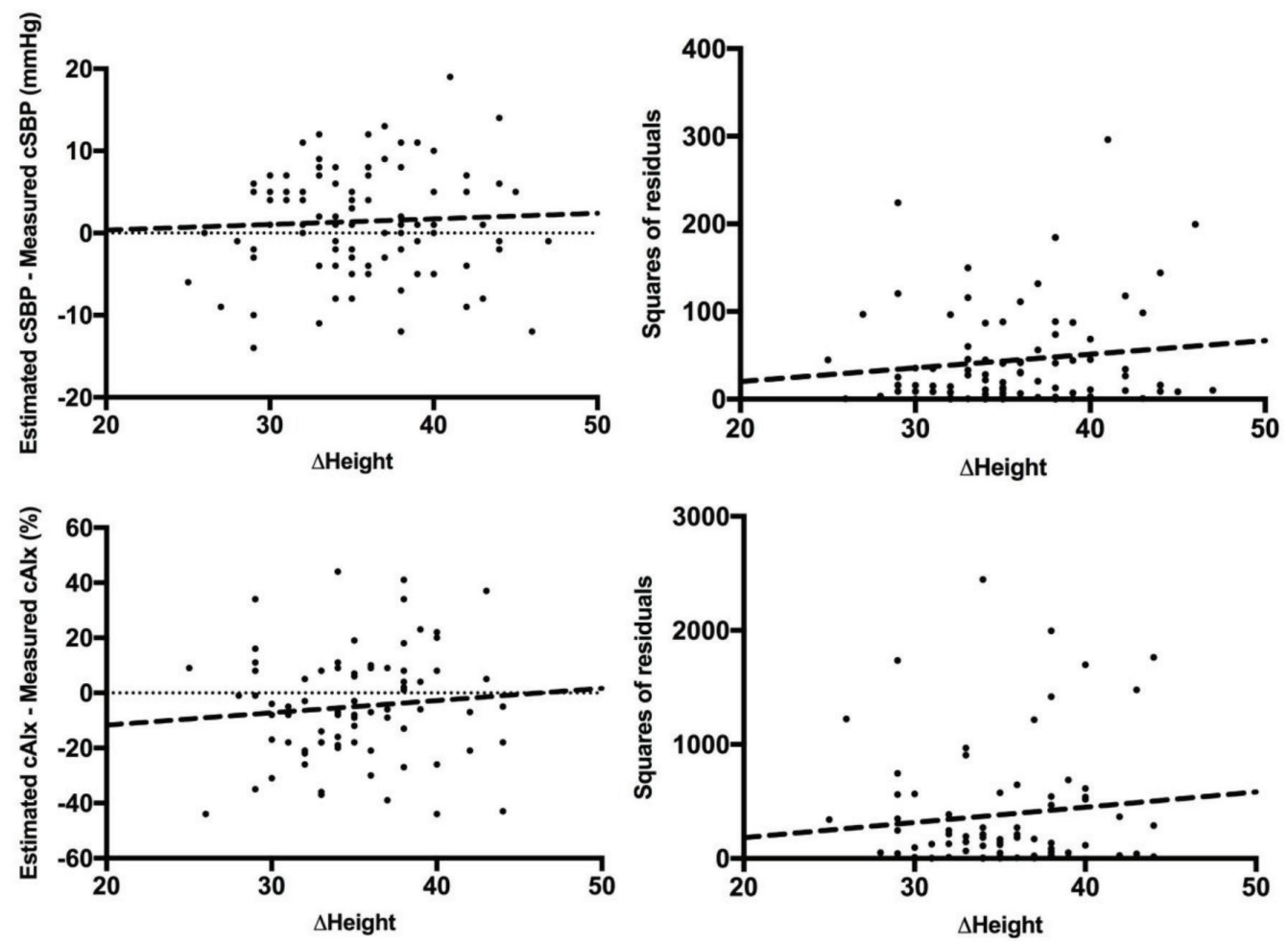

Figure 1 Change in height between age 8 and 14 years, versus the accuracy and precision of iTF-estimated cSBP and cAIx at 14 years of age. Modelling error (accuracy) for cSBP against change in height (top-left) and the squared residuals of this modelling error (precision) as a function of change in height (top-right). Modelling error for cAIx against change in height (bottom-left) and the squared residuals of this modelling error as a function of change in height (bottom-right).
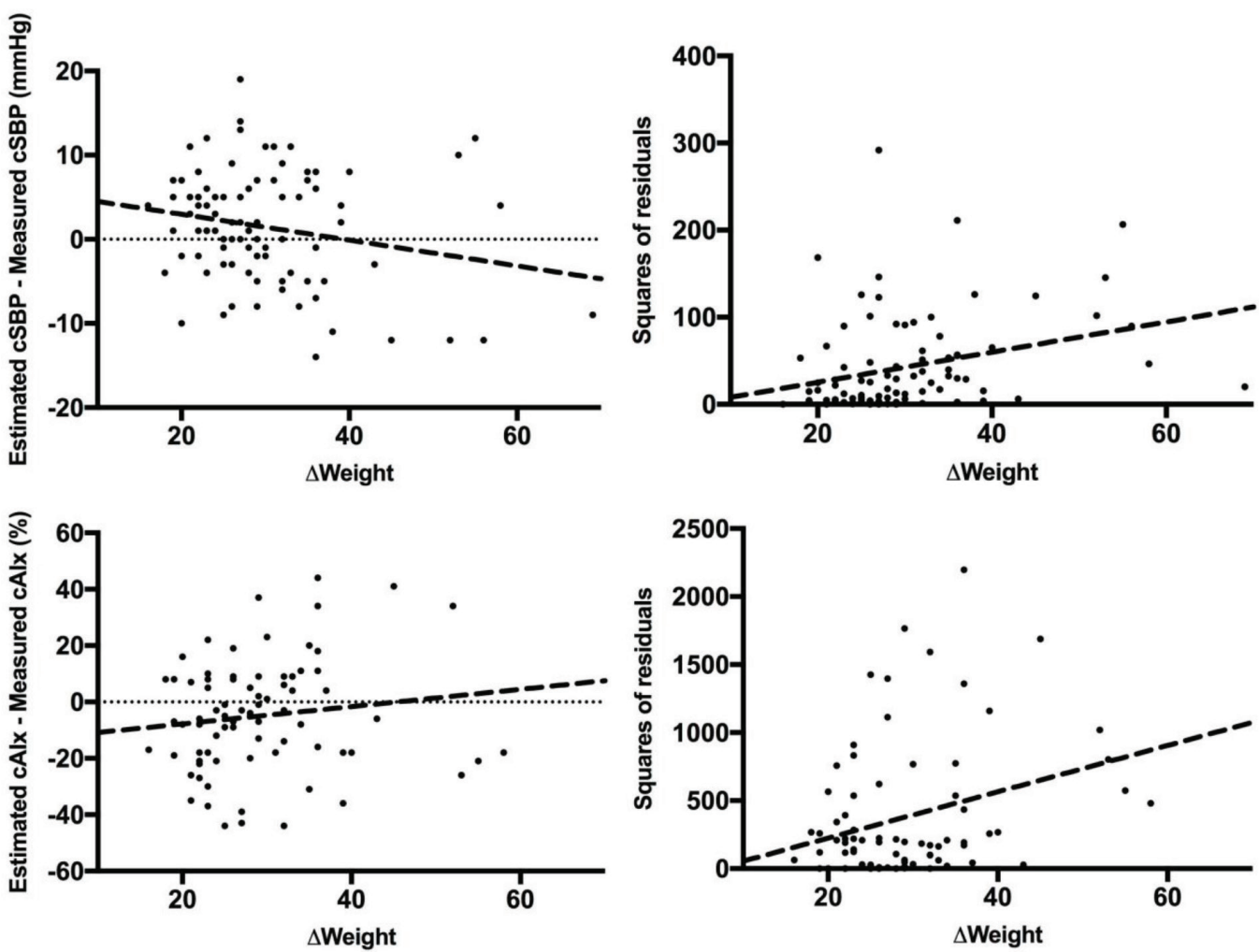

Figure 2 Change in weight between age 8 and 14 years, versus the accuracy and precision of iTF-estimated cSBP and cAIx at 14 years of age. Modelling error (accuracy) for cSBP against change in weight (top-left) and the squared residuals of this modelling error (precision) as a function of change in weight (top-right). Modelling error for cAIx against change in weight (bottom-left) and the squared residuals of this modelling error as a function of change in weight (bottom-right). 
properties of the vascular tree and wave reflection properties. Although these changes are qualitatively expected, due to the rapid changes of in arterial path length during childhood growth, this unique longitudinal study is able to provide quantitative comparisons.

The basis for upper limb assessment for synthesizing central aortic waveforms is predicated on the minimally changing wave transmission properties of the upper limb with age, disease and drug therapy in adults [22]. To the knowledge of these authors, there are currently no published longitudinal studies in adults or children that attempt to assess performance of radial-to-central transfer functions or their intra-individual validity over time. As such, these above assumptions of minimal change in upper limb vasculature cannot be conclusively ascribed to children and it is unknown whether the extension and widening of major vasculature concurrent to changes in the elasticity of the conduit vessels during this growth period within and between children may adversely affect the predictive accuracy of iTFs.

One notable association we found in this study was that height and pSBP only became apparent in later adolescence, and that while taller adolescents had higher average pSBP, this is not similarly the case for cSBP. That change in height is not associated with this increase in pSBP nor measured cSBP, while change in weight is, suggesting that perhaps baseline blood pressure may be determined by an individual's potential for vertical growth and final height, but potentially further influenced by obesity-associated hemodynamic changes [23]. We also note that despite boys growing taller and gaining more BMI between the ages of 8 and 14 years, it is the change in weight, especially in boys, that has the greater effect on the accuracy and precision of cSBP and cAIx estimates. These results suggest that the sex of the growing child also affects the characteristics of a developing vascular system, and perhaps should be accounted for in central hemodynamic modelling.

The decrease in predictive accuracy and precision of the iTFs at 14 -years of age, compared to 6 years prior at the time of development, suggests that they may be affected by one or more aspects of growth between these two time-points. We demonstrate that an increase in weight is associated with a decrease in iTF accuracy and precision for predicting $\mathrm{CSBP}$, and also the precision but not accuracy for estimating cAIx. Increases in BMI and waist circumference were similarly associated with decreased precision in CSBP only. Decrease in HR between 8 and 14 years of age however, was found only to be associated with decreased precision of iTF to estimate cAIx. Changes in height on the other hand did not appear to influence iTF accuracy.

Essentially, this analysis aims to determine the need for age-appropriate TFs for the assessment of central arterial hemodynamics in children, given the heterogeneity between children and along the time-course of adolescent growth. In adults, the robustness of gTFs has been interrogated under a number of different hemodynamic conditions, such as under stress of pharmacological and exercise stimuli, with results suggesting that the gTF methodology continue to perform robustly under these differing conditions [10,20,24]. Extrapolating these findings, it can be presumed that a gTF that performs well at rest in a child would similarly do so under dynamic conditions. A recent study by Mynard et al. [25] looking at central blood pressure estimation in children using the SphygmoCor adult gTF demonstrates excellent accuracy when compared to invasively obtained pressure data at rest when correctly calibrated for mean and diastolic pressures. Contrastingly, wave reflection and augmentation index was found to be poorly estimated using these same methods [26].

Firstly, regarding the absolute accuracy of the iTF method for estimating central hemodynamic parameters, we first note that at both 8 and 14 years of age, the iTF estimates cSBP within the validation criteria proposed by the ARTERY society of $5 \pm 8 \mathrm{mmHg}$ as adapted from the AAMI standard [21,27]. Contrastingly, there is no consensus regarding validation criteria for CAIx measurements. For perspective, Mynard et al. [26], in comparing non-invasive to invasive estimates of cAIx in children reported a mean absolute overestimation of $34.2 \%$ with non-invasive cuff-based SphygmoCor XCEL device (AtCor Medical). In adults, Chen et al. [10] found the gTF-derived cAIx was on average an absolute $7 \%$ lower than when invasively measured. An absolute error in iTF-derived cAIx of 5.2\% can hence be considered relatively reasonable. It should be noted however, that the aforementioned studies involved invasively-obtained invasive waveforms, whereas the use of a non-invasive carotid artery waveform as a surrogate does confound direct comparisons.

For an alternative comparison, we previously developed ageappropriate gTFs in children based on non-invasive arterial waveform data, and reported that an age-appropriate gTF developed in a cohort of 50 8-year-old children had an accuracy within $2.1 \pm 4.4$ $\mathrm{mmHg}$ for estimating cSBP and $-6.6 \pm 11.9 \%$ for estimating cAIx in a similar profile cohort of 8-year-old children, who were also part of the CardioCAPs study. This is both less accurate and precise than the iTFs in this study, as expected. When this same gTF was applied in an independent cohort of 14-year-old children, it estimated cSBP and cAIx within $4.9 \pm 6.1 \mathrm{mmHg}$ and $-8.4 \pm 18.0 \%$ respectively, again less accurate than the iTFs reported in this study analysis [28]. From these results, it can be seen that the iTF-derived parameters used in this study are both absolutely and relatively accurate in estimating central hemodynamic parameters.

A number of epidemiological studies have used adult-validated devices to establish cSBP and cAIx references values in children [29-32]. Furthermore, ongoing hemodynamic research in the young has led to promising developments for a potential role of central hemodynamic indices as tools for cardiovascular risk stratification or markers of early disease [33-35]. As such, the need for paediatric validation of the gTF methodology for estimated central arterial hemodynamics, as well as for other devices such as the Mobil-O-graph (IEM GmbH; Stolberg; Germany) and Arteriograph (TensioMed; Budapest, Hungary) systems, is certainly present and must be addressed.

There are a few notable limitations to these analyses. Firstly, there are a relatively small number of participants $(n=91)$. This was a retrospective analysis, from which paired longitudinal waveform data was only available for a relatively small subset of participants. As such, our conclusions may be prone to type II error. Secondly, the short-term accuracy of iTFs (i.e. repeat measurements within days to weeks) is unknown from these data, and as such cannot inform between the reproducibility of the technique and true changes over 6 -years secondary to growth and physiological change. Finally, the use of carotid artery tonometry to non-invasively measure central aortic waveforms in children has not been rigorously evaluated previously. Although this has been demonstrated to be a reasonable 
surrogate for central aortic waveforms in adults, how well this methodology performs for central hemodynamic assessment in children is unknown [12]. Lack of gold-standard invasive central aortic waveform data for this study remains one major limitation, but it is not appropriate to apply invasive catheterization in healthy children without clinical indication. Nevertheless, radial-to-carotid TFs function similarly to radial-to-aortic TFs, and serve as a representative model of the vasculature for the purposes of this study. Furthermore, we have not accounted for changes in pulse wave velocity, given the likelihood of collinearity. This may be a topic for future studies given that height, age, body weight and blood pressure are all determinants of pulse wave velocity in children, and changes in pulse wave velocity may impact the accuracy of cSBP and cAIx predication from individualized hemodynamic modelling.

\section{CONCLUSION}

Changes in heart rate and body habitus during childhood and adolescence, in particular weight and BMI, are associated with concurrent changes in the predictive accuracy and precision of iTFs. These data suggest that the frequency response characteristics of a person's vascular system may be affected by changes in these parameters, which may need to be considered and accounted for when developing and applying generalized TFs for use in a heterogenous pediatric population.

\section{CONFLICTS OF INTEREST}

AQ is an employee of AtCor Medical, Sydney, Australia. No potential conflict of interest was reported by the remaining authors.

\section{AUTHORS' CONTRIBUTION}

TC planned the study, organised and processed the data, performed statistical analyses, and drafted the manuscript. AQ, MB, JA and MS provided intellectual contributions study design and interpretation of data. All authors contributed to revision of the manuscript.

\section{ACKNOWLEDGMENTS}

This work was supported by a NSW CVRN Research Development Project Grant (\#101135) from the National Heart Foundation of Australia; Cooperative Research Centre for Asthma, Department of Health, Children's Hospital at Westmead, and National Health and Medical Research Council of Australia Project and Program Grants (\#222722; \#482800); and The Financial Markets Foundation for Children grant (\#2012-114). MRS was supported by a National Heart Foundation of Australia Future Leader Fellowship (100419).

\section{SUPPLEMENTARY MATERIALS}

Supplementary data related to this article can be found at https:// doi.org/10.2991/artres.k.201003.001.

\section{REFERENCES}

[1] Vos T, Barber RM, Bell B, Bertozzi-Villa A, Biryukov S, Bolliger I, et al. Global, regional, and national incidence, prevalence, and years lived with disability for 301 acute and chronic diseases and injuries in 188 countries, 1990-2013: a systematic analysis for the Global Burden of Disease Study 2013. Lancet 2015;386:743-800.

[2] Chen X, Wang Y. Tracking of blood pressure from childhood to adulthood. Circulation 2008;117:3171-80.

[3] Camhi SM, Katzmarzyk PT. Tracking of cardiometabolic risk factor clustering from childhood to adulthood Int J Pediatr Obes 2010;5:122-9.

[4] Juonala M, Cheung MMH, Sabin MA, Burgner D, Skilton MR, Kähönen M, et al. Effect of birth weight on life-course blood pressure levels among children born premature: the cardiovascular risk in Young Finns Study. J Hypertens 2015;33:1542-8.

[5] Zanetti D, Tikkanen E, Gustafsson S, Priest JR, Burgess S, Ingelsson E. Birthweight, type 2 diabetes mellitus, and cardiovascular disease: addressing the barker hypothesis with Mendelian randomization. Circ Genom Precis Med 2018;11:e002054.

[6] Roman MJ, Devereux RB, Kizer JR, Lee ET, Galloway JM, Ali T, et al. Central pressure more strongly relates to vascular disease and outcome than does brachial pressure: the Strong Heart Study. Hypertension 2007;50:197-203.

[7] Roman MJ, Devereux RB, Kizer JR, Okin PM, Lee ET, Wang W, et al. High central pulse pressure is independently associated with adverse cardiovascular outcome. The Strong Heart Study. J Am Coll Cardiol 2009;54:1730-4.

[8] Vlachopoulos C, Aznaouridis K, Stefanadis C. Prediction of cardiovascular events and all-cause mortality with arterial stiffness: a systematic review and meta-analysis. J Am Coll Cardiol 2010;55:1318-27.

[9] Mitchell GF, Hwang SJ, Larson MG, Hamburg NM, Benjamin EJ, Vasan RS, et al. Transfer function-derived central pressure and cardiovascular disease events: the Framingham Heart Study. J Hypertens 2016;34:1528-34.

[10] Chen CH, Nevo E, Fetics B, Pak PH, Yin FC, Maughan WL, et al. Estimation of central aortic pressure waveform by mathematical transformation of radial tonometry pressure: validation of generalized transfer function. Circulation 1997;95:1827-36.

[11] Hope SA, Tay DB, Meredith IT, Cameron JD. Use of arterial transfer functions for the derivation of aortic waveform characteristics. J Hypertens 2003;21:1299-305.

[12] Segers P, Rietzschel E, Heireman S, De Buyzere M, Gillebert T, Verdonck P, et al. Carotid tonometry versus synthesized aorta pressure waves for the estimation of central systolic blood pressure and augmentation index. Am J Hypertens 2005; 18:1168-73.

[13] Cheng HM, Chuang SY, Sung SH, Wu CC, Wang Jr J, Hsu PF, et al. 2019 Consensus of the Taiwan Hypertension Society and Taiwan Society of Cardiology on the clinical application of central blood pressure in the management of hypertension. Acta Cardiol Sin 2019;35:234-43.

[14] Wang S, Zhou Z, Fan F, Qi L, Jia J, Sun P, et al. Joint effect of non-invasive central systolic blood pressure and peripheral systolic blood pressure on incident hypertension in a chinese community-based population. Sci Rep 2018;8:3229. 
[15] Sharman JE, Marwick TH, Gilroy D, Otahal P, Abhayaratna WP, Stowasser M, et al. Randomized trial of guiding hypertension management using central aortic blood pressure compared with best-practice care. Hypertension 2013;62:1138-45.

[16] Hope SA, Meredith IT, Tay D, Cameron JD. 'Generalizability' of a radial-aortic transfer function for the derivation of central aortic waveform parameters. J Hypertens 2007;25:1812-20.

[17] Hahn JO. Individualized estimation of the central aortic blood pressure waveform: a comparative study. IEEE J Biomed Health Inform 2014; 18:215-21.

[18] Mihrshahi S, Peat JK, Webb K, Tovey ER, Marks GB, Mellis CM, et al. The childhood asthma prevention study (CAPS): design and research protocol of a randomized trial for the primary prevention of asthma. Control Clin Trials 2001;22:333-54.

[19] Jones CR, Taylor K, Poston L, Shennan AH. Validation of the Welch Allyn 'Vital Signs' oscillometric blood pressure monitor. J Hum Hypertens 2001;15:191-5.

[20] Pauca AL, O'Rourke MF, Kon ND. Prospective evaluation of a method for estimating ascending aortic pressure from the radial artery pressure waveform. Hypertension 2001;38:932-7.

[21] Sharman JE, Avolio AP, Baulmann J, Benetos A, Blacher J, Leigh Blizzard C, et al. Validation of non-invasive central blood pressure devices: ARTERY Society task force consensus statement on protocol standardization. Eur Heart J 2017;38:2805-12.

[22] Karamanoglu M, O’Rourke MF, Avolio AP, Kelly RP. An analysis of the relationship between central aortic and peripheral upper limb pressure waves in man. Eur Heart J 1993;14:160-7.

[23] Katz SH, Hediger ML, Schall JI, Bowers EJ, Barker WF, Aurand $\mathrm{S}$, et al. Blood pressure, growth and maturation from childhood through adolescence. Mixed longitudinal analyses of the Philadelphia Blood Pressure Project. Hypertension 1980; 2:55-69.

[24] Sharman JE, Lim R, Qasem AM, Coombes JS, Burgess MI, Franco J, et al. Validation of a generalized transfer function to noninvasively derive central blood pressure during exercise. Hypertension 2006;47:1203-8.

[25] Mynard JP, Goldsmith G, Springall G, Eastaugh L, Lane GK, Zannino D, et al. Central aortic blood pressure estimation in children and adolescents: results of the KidCoreBP study. J Hypertens 2020;38:821-8.
[26] Mynard J, Goldsmith G, Kowalski R, Eastaugh L, Lane G, Springall $\mathrm{G}$, et al. Quantifying wave reflection in children: invasive vs non-invasive central augmentation index and reflection magnitude and their association with left ventricular mass. Artery Res 2018;24:92.

[27] American National Standard. Non-invasive Sphygmomanometers - Part 2: clinical validation of automated measurement type. ANSI/AAMI/ISO 81060-2:2009. Arlington, Virginia: Association for the Advancement of Medical Instrumentation; 2009.

[28] Cai TY, Qasem A, Ayer JG, Butlin M, Meagher SO, Melki $\mathrm{C}$, et al. Central blood pressure in children and adolescents: non-invasive development and testing of novel transfer functions. J Hum Hypertens 2017;31:831-7.

[29] Hidvégi EV, Illyés M, Molnár FT, Cziráki A. Influence of body height on aortic systolic pressure augmentation and wave reflection in childhood. J Hum Hypertens 2014;29:495-501.

[30] Elmenhorst J, Hulpke-Wette M, Barta C, Pozza RD, Springer S, Oberhoffer R. Percentiles for central blood pressure and pulse wave velocity in children and adolescents recorded with an oscillometric device. Atherosclerosis 2015;238:9-16.

[31] Diaz A, Zócalo Y, Bia D, Fischer ED. Reference intervals of central aortic blood pressure and augmentation index assessed with an oscillometric device in healthy children, adolescents, and young adults from Argentina. Int J Hypertens 2018;2018:1469651.

[32] Kahn FK, Wake M, Lycett K, Clifford S, Burgner DP, Goldsmith $\mathrm{G}$, et al. Vascular function and stiffness: population epidemiology and concordance in Australian children aged 11-12 years and their parents. BMJ Open 2019;9:34-43.

[33] Litwin M, Obrycki Ł, Niemirska A, Sarnecki J, Kułaga Z. Central systolic blood pressure and central pulse pressure predict left ventricular hypertrophy in hypertensive children. Pediatr Nephrol 2019;34:703-12.

[34] Alves C, Pinho JF, dos Santos LM, Magalhães G, da Silva JM, Fontes FL, et al. Augmentation index, a predictor of cardiovascular events, is increased in children and adolescents with primary nephrotic syndrome. Pediatr Nephrol 2020;35:815-27.

[35] Faconti L, Silva MJ, Molaodi OR, Enayat ZE, Cassidy A, Karamanos A, et al. Can arterial wave augmentation in young adults help account for variability of cardiovascular risk in different British ethnic groups? J Hypertens 2016;34:2220-6. 